\title{
An Integrated GNSS/LiDAR-SLAM Pose Estimation Framework for Large-Scale Map Building in Partially GNSS-Denied Environments
}

\author{
Guojian $\left.\mathrm{He}^{(}\right)$, Xingda Yuan ${ }^{\circledR}$, Yan Zhuang ${ }^{\circledR}$, Member, IEEE, and Huosheng $\mathrm{Hu}^{\circledR}$, Senior Member, IEEE
}

\begin{abstract}
This article presents an integrated global navigation satellite system/light detection and ranging (GNSS/LiDAR)-based simultaneous localization and mapping (SLAM) pose estimation framework to perform large-scale 3-D map building in partially GNSS-denied outdoor environments. The framework takes the advantage of the complementarity between GNSS positioning and LiDAR-SLAM to decompose the map building task according to the GNSS real-time kinematic (RTK) status. When mapping in GNSS-denied scenes, a 3-D LiDAR-SLAM algorithm is adopted to estimate poses and a correction algorithm is presented to correct drift errors. On the other hand, when mapping in open scenes, a GNSS-initialized LiDAR mapping algorithm (GL-mapping) is proposed to loosely couple GNSS positioning and LiDAR data registration. It can perform the orientation estimation without the use of either the high-cost inertial sensing device or the GNSS dual-antenna. Experiments are conducted in large-scale outdoor environments to demonstrate that the proposed framework can accomplish simultaneous pose estimation and map building with high precision in both open scenes and GNSS-denied scenes.
\end{abstract}

Index Terms-Global navigation satellite system (GNSS)denied environments, large-scale map building, multisensor data acquisition, pose estimation.

\section{INTRODUCTION}

A UTONOMOUS 3-D map building is a crucial ability for mobile robots or mobile laser scanning (MLS) systems working in large-scale outdoor environments [1], [2]. The quality of data for map building (the registered 3-D point clouds) is related to the accuracy of the pose estimation algorithm. Typically, the pose data are provided by the data fusion between the global navigation satellite system (GNSS) and the inertial navigation system (INS) in outdoor environments [3]-[5]. The GNSS/INS system can achieve accurate 6-DoF pose data (3-DoF position and 3-DoF orientation) with the help of a real-time kinematic (RTK) technique.

Manuscript received July 19, 2020; accepted September 3, 2020. Date of publication September 17, 2020; date of current version December 4, 2020. This work was supported by the National Natural Science Foundation of China under Grant U1913201. The Associate Editor coordinating the review process was George Xiao. (Corresponding author: Yan Zhuang.)

Guojian He, Xingda Yuan, and Yan Zhuang are with the School of Control Science and Engineering, Dalian University of Technology, Dalian 116024, China (e-mail: hgjmoon@mail.dlut.edu.cn; yxd0110@mail.dlut.edu.cn; zhuang@dlut.edu.cn).

Huosheng $\mathrm{Hu}$ is with the School of Computer Science and Electronic Engineering, University of Essex, Colchester CO4 3SQ, U.K. (e-mail: hhu@essex.ac.uk).

Digital Object Identifier 10.1109/TIM.2020.3024405
However, the orientation data from the GNSS/INS system usually need the support of the dual-antenna, especially for the real-world applications where magnetometer suffers from electromagnetic interference. Therefore, the dual-antenna is necessary for absolute orientation measurement, but its mechanical installation size (recommended baseline length is greater than $2 \mathrm{~m}$ ) makes it unsuitable for portable MLS systems or mobile robot platforms. Moreover, there are many GNSS-denied scenes in outdoor environments due to the occlusion and reflection of tall trees and buildings. As the RTK positioning data are not available in these places, the GNSS/INS systems require strongly high-cost inertial devices to improve their localization accuracy.

Light detection and ranging (LiDAR)-based simultaneous localization and mapping (SLAM) is another suitable solution to perform 3-D map building with high accuracy, especially for the GNSS-denied scenes [6]-[10]. Several commercial products based on the LiDAR-SLAM solution have been developed mainly for mapping in indoor environments [11]-[13]. The LiDAR-SLAM-based MLS systems are generally backpack-mounted and suitable for single human operation, namely the personal MLS systems, which do not need GNSS data and can achieve real-time mapping results with centimeter-level accuracy while working in indoor scenes. However, the SLAM-based systems suffer from accumulated drift errors and computational failures in large-scale outdoor scenes with sparse geometrical features.

Recently, researchers have investigated how to fuse GNSS positioning and LiDAR-SLAM for building large-scale outdoor environment maps. The fusion methods can take advantage of the complementarity of different pose estimation methods: GNSS positioning performs well in open scenes, while the LiDAR-SLAM is more suitable in GNSSdenied scenes. In [14]-[17], the classic fusion methods were implemented under the probabilistic framework of Kalman filtering in order to provide more robust pose estimation results than the method that is only based on GNSS positioning or LiDAR-SLAM. However, most of the aforementioned fusion methods need GNSS dual-antenna to achieve accurate orientation measurements, which limits the applications on small-sized unmanned systems. In [10], a LiDAR-SLAM system LIO-SAM is proposed, in which a factor graph is applied to incorporate the 3-DoF GNSS positioning data as factors into the system. However, LIO-SAM is a typical SLAM framework, in which the coordinate system between GNSS and 
LiDAR is not accurately calibrated and the GNSS positioning data are not fully utilized in open scenes. To the author's knowledge, some of the commercial MLS products (e.g., Leica's Pegasus-Backpack ${ }^{1}$ and Kaarta's stencil-pro ${ }^{2}$ ) have recently integrated GNSS positioning into their SLAM-based systems, but no academic literature can be found for the details of the system implementation and test evaluation.

In this article, we will focus on developing a mobile mapping system operated in large-scale outdoor environments. In order to achieve easy deployment on small robot platforms or personal MLS systems, the developed system does not require the high-cost inertial device or the GNSS dual-antenna. An integrated GNSS/LiDAR-SLAM pose estimation framework is proposed to perform 3-D map building in partially GNSS-denied environments. The proposed framework takes advantage of the complementarity between GNSS positioning and LiDAR-SLAM. Unlike the existing methods, the map building task in our work is decomposed into GNSS-LiDAR mapping mode in open scenes (where the RTK positioning is available) and LiDAR-only mapping mode in GNSS-denied scenes.

When working in the GNSS-LiDAR mapping mode, the 3-DoF positions are available from GNSS RTK positioning. However, the 3-DoF orientations are unavailable for the reason that the high-cost inertial device and the GNSS dual-antenna cannot be used for the portable hardware system. To solve this problem, the GNSS-initialized LiDAR mapping algorithm (GL-mapping) is proposed to estimate orientations without orientation measurements from the hardware. GL-mapping is an optimization-based algorithm that loosely couples GNSS positioning and LiDAR data registration.

When working in the LiDAR-only mapping mode, a stateof-the-art 3-D LiDAR-SLAM algorithm is adopted to estimate poses and perform map building in GNSS-denied scenes. A graph-based optimization algorithm is utilized to correct the drift errors of the LiDAR-SLAM algorithm. However, the graph-based correction algorithm is sensitive to the initial pose values. We propose a backward-adjustment method to adjust the initial pose values which can improve the performance of the graph-based optimization.

The novelty and contributions of our work are concluded as follows:

1) An integrated pose estimation framework is proposed and implemented for both mapping in open scenes and mapping in GNSS-denied scenes.

2) For mapping in open scenes, an optimization-based algorithm is proposed to solve the problem of estimating orientations without the support of the high-cost inertial device or the GNSS dual-antenna, which loosely couples GNSS positioning and LiDAR data registration.

3) Engineering contributions include the extendable hardware implementation with hard synchronization and the driver code for its multisensor data acquisition. ${ }^{3}$ The

\footnotetext{
${ }^{1}$ https://leica-geosystems.com/products/mobile-sensor-platforms/captureplatforms/leica-pegasus-backpack

${ }^{2}$ https://www.kaarta.com/products/stencil-pro/

${ }^{3}$ https://github.com/ZhuangYanDLUT/hard_sync_dut
}

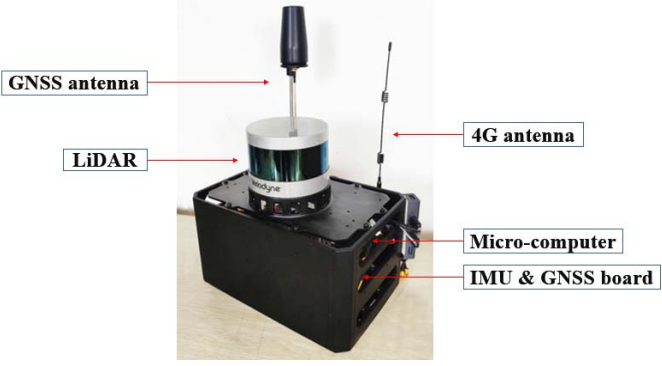

Fig. 1. Hardware composition of 3D-M-Box.

source codes of the 3-D map building framework are also made available online. ${ }^{4}$

The rest of this article is organized as follows. Section II shows the hardware implementation. Section III describes the details of the 3-D map building framework. In Section IV, we test the proposed methods with several groups of field experiments. Finally, a brief conclusion and future work are given in Section V.

\section{IMPLEMENTATION OF HARDWARE SYSTEM}

A typical multisensor data acquisition system named 3D-M-Box is developed in this article, which integrates LiDAR, GNSS, and inertial measurement unit (IMU) for 3-D map building in partially GNSS-denied environments. We have used the off-the-shelf hardware components and built an efficient solution to achieve high precision, portability, extendibility, and hard synchronization. The readers can easily reproduce a similar mapping system according to this article. In the rest of this article, 3D-M-Box is applied as the hardware platform to describe and execute the proposed algorithms.

Fig. 1 shows the detailed hardware components of 3D-M-Box, which includes a Velodyne VLP-16 LiDAR, a NovAtel OEM718-D GNSS board, and an Xsens Mti-30 IMU. A micro-computer is also integrated for online computation, which is equipped with Intel i7-8550U CPU, 8-GB RAM, and 512-GB SSD. The Xsens IMU provides a prior estimation for the LiDAR-SLAM algorithm. The RTK data are imported from Qianxun SI by a built-in 4G module and its output frequency is $5 \mathrm{~Hz}$. Though dual-antenna can be supported by the integrated GNSS board, a single antenna is deployed to make the system portable and flexible. It should be noted that the hardware system of 3D-M-Box can be easily extended with other perception sensors such as binocular cameras.

Instead of using the computer system time for software synchronization, we use hard synchronization that is naturally more precise for timestamp acquisition of multisensor data. Fig. 2 shows our hard-synchronization framework, which makes full use of the electrical characteristics of Xsens IMU and GNSS board. Velodyne LiDAR is synchronized with GNSS pulse per second (PPS). Other perception sensors (e.g., cameras) with trigger functions can be triggered by the SyncOut pin of the Xsens IMU, and IMU is synchronized by the

\footnotetext{
${ }^{4}$ https://github.com/ZhuangYanDLUT/lidar_gnss_mapping
} 


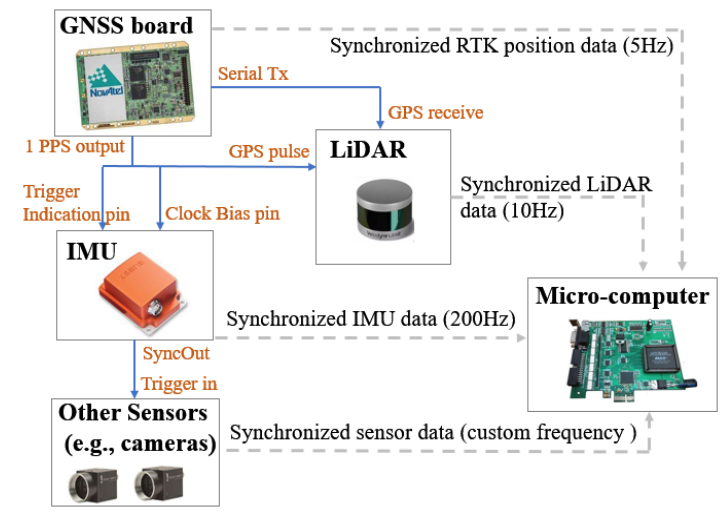

Fig. 2. Framework for hard-synchronization of multiple sensors.

GNSS PPS signal. The whole system clock is in accordance with the PPS signal. The detailed electrical protocol can be seen from the Velodyne user manual ${ }^{5}$ and the Xsens Mti user manual. ${ }^{6}$ Simultaneously, the system software driver must be implemented carefully to deal with problems such as the absence of PPS signal, the transmission delay, and data index association for multisensor data acquisition. More details can be seen in our source code online.

\section{System FRAMEWORK FOR 3-D MAP BUILDING}

Fig. 3(a) and (b) presents the integrated GNSS/ LiDAR-SLAM pose estimation framework for large-scale 3-D map building in partially GNSS-denied scenes. Our proposed framework consists of two operating modes: GNSS-LiDAR mapping mode in open scenes and LiDAR-only mapping mode in GNSS-denied scenes. It is inspired by the fact that GNSS positioning with RTK is accurate enough for map building in open scenes, while LiDAR-SLAM is suitable in GNSS-denied scenes.

When working in GNSS-denied scenes, 3D-M-Box runs in the LiDAR-only mapping mode. The RTK positioning data are unavailable and a state-of-the-art 3-D LiDAR-SLAM algorithm is adopted to perform 3-D map building. However, there are increasing accumulative errors that can only be corrected by the RTK positioning data when 3D-M-Box switches to GNSS-LiDAR mapping mode. The drift error of the pose estimation is eliminated by a Drift Error Correction module and the corrected output data of poses and point clouds will be provided [see Fig. 3(a)].

When working in open scenes, the RTK positioning data are available and 3D-M-Box runs in the GNSS-LiDAR mapping mode. We present an optimization-based algorithm GL-mapping to loosely couple GNSS positioning and the LiDAR data registration for estimating positions as well as orientations. GL-mapping consists of two major modules: auto coordinate alignment and GNSS-initialized LiDAR registration. The auto coordinate alignment module performs an online calibration step, which aligns the coordinate between LiDAR and GNSS by iteratively registering GNSS RTK positions and the feedback positions of final estimated poses (the positions

\footnotetext{
${ }^{5}$ https://www.velodyneLiDAR.com/downloads.html

${ }^{6} \mathrm{https}: / /$ content.xsens.com/mti-10-manual
}

from LiDAR-SLAM algorithm are used when there are no feedback positions at the initial phase). On the other hand, the final estimated poses and point clouds are generated by the GNSS-initialized LiDAR registration module with the unified coordinate system [see Fig. 3(b)].

We now define notations and frame definitions that we use throughout this article. LiDAR coordinate system $\{L\}$ is a local coordinate system with its origin at the geometric center of the LiDAR. The results of LiDAR-SLAM are in a relative coordinate system $\{S\}$ coinciding with $\left\{L_{0}\right\}$ (i.e., $\{L\}$ at the initial pose). The GNSS positioning data are defined in an absolute North-East-Down coordinate system which is denoted as the world coordinate system $\{W\}$. The purpose of the proposed pose estimation framework is to estimate the transformation $\mathbf{T}^{W}$ in $\{W\}$ as 3D-M-Box moves in partially GNSS-denied environments (the point cloud $P^{W}$ can be calculated by $\mathbf{T}^{W}$ directly). The transformation $\mathbf{T}$ can be formulated as $(\boldsymbol{\theta}, \mathbf{t})$ or $(\mathbf{R}, \mathbf{t})$, where $\mathbf{t}$ is the 3 -DoF position, $\boldsymbol{\theta}$ is the $3-\mathrm{DoF}$ orientation (i.e., yaw, pitch, and roll), and $\mathbf{R}$ is the corresponding rotational matrix.

\section{A. LiDAR-Only Mapping}

We adopt the state-of-the-art LiDAR-SLAM algorithm laser odometry and mapping (LOAM) in the LiDAR-only mapping mode, which was proven to be efficient for mapping in large-scale outdoor environments with acceptable precision [7]. Other existing algorithms need particular laser points of the ground [8] or indispensable IMU data in a tightly coupled manner [9], [10], which cannot meet the demand for various extendibility of the mapping system. GNSS-denied scenes are places with tall buildings and trees, where geometrical features are abundant for the LOAM algorithm. Modifications are conducted to initialize the coordinate system of the LOAM algorithm from $\{S\}$ to $\{W\}$ with the last estimated pose of former GNSS-LiDAR mapping mode. The initialization of the coordinate system is performed every time 3D-MBox switches to LiDAR-only mapping mode from GNSSLiDAR mapping mode.

The accumulative drift errors of the LOAM algorithm cannot be ignored in the LiDAR-only mapping mode, especially for long-distance travel. As is shown in Fig. 4, the drift errors result in a gap between the last estimated pose $\mathbf{T}_{\mathrm{L}}$ of LiDAR-only mapping mode and the first estimated pose $\mathbf{T}_{\mathrm{G}}$ of GNSS-LiDAR mapping mode. It should be noted that the poses mentioned in this section are all in $\{W\}$ and the superscript is omitted for convenience. In our work, the Drift Error Correction module with graph optimization-based iSAM2 [18] algorithm is utilized to fill the gap. Noted that $\mathbf{T}_{\mathrm{G}}$ has no drift errors with the constraint of GNSS RTK positioning, which is regarded as the ground-truth control point for iSAM2. The poses that belong to the whole estimated trajectory of LOAM in LiDAR-only mapping mode will be optimized to eliminate the drift errors.

It was found, however, that iSAM2 usually obtained bad results in some conditions, where the optimized trajectory was in distortion. The reason is that the graph model in this work has only one constraint, that is, the constraint of $\mathbf{T}_{\mathrm{L}}$ and $\mathbf{T}_{\mathrm{G}}$ 


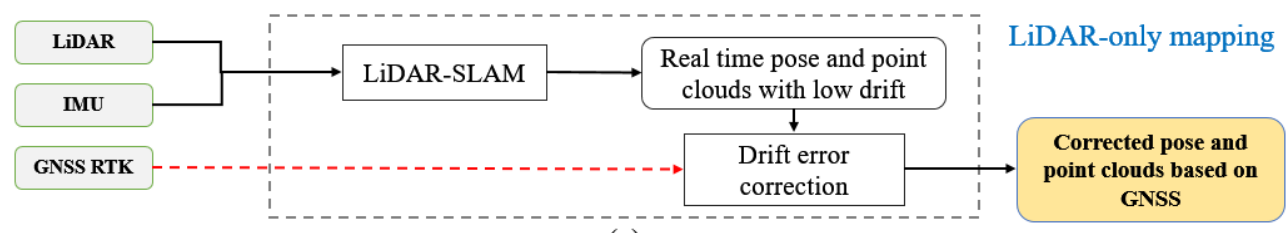

(a)

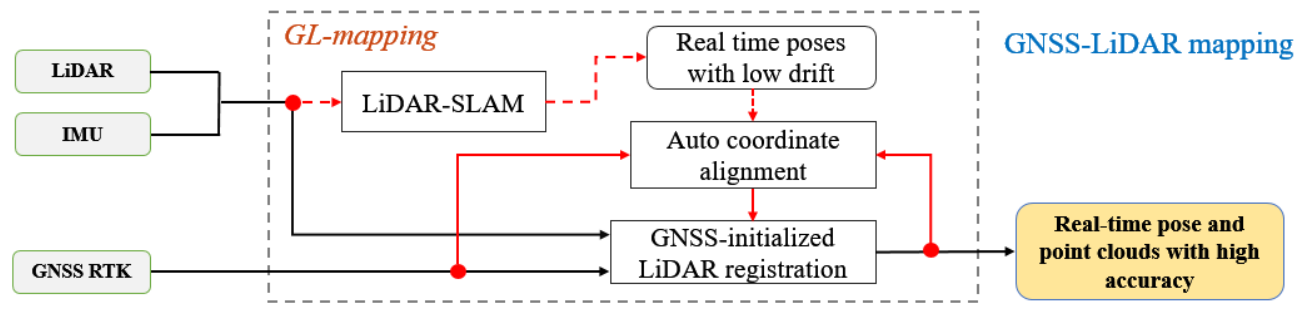

(b)

Fig. 3. Overall framework of the large-scale 3-D map building system switching between (a) LiDAR-only mapping mode and (b) LiDAR-GNSS mapping mode in partially GNSS-denied scenes. The solid arrow with black color describes the continuous data flow. The solid arrow with red color describes the data flow sustaining for a period of time (e.g., until the particular data result converges). The dotted arrow with red color describes that the data flow occurs only once or for a short time (e.g., until the particular initialization finishes).

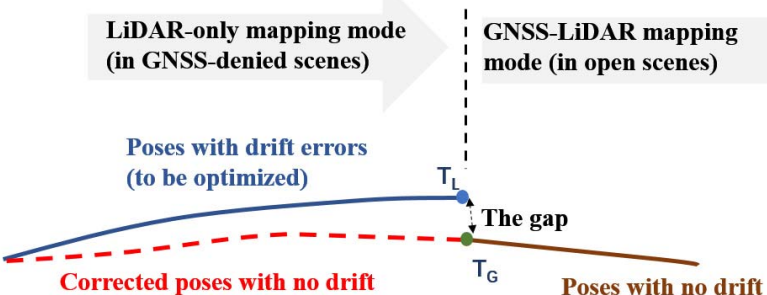

Fig. 4. Illustration of drift errors in LiDAR-only mapping mode.

in Fig. 4, and the optimization becomes very sensitive with initial values of the poses. To provide an accurate initialization for iSAM2, we propose a backward-adjustment method in the Drift Error Correction module to equally distribute the final gap error backwardly to the poses along the whole trajectory before the graph optimization step.

The proposed backward-adjustment method adjusts 3-DoF positions and 3-DoF orientations, respectively. Assuming that $\mathbf{T}_{1}, \mathbf{T}_{2}, \ldots, \mathbf{T}_{n}$ are the set of $n$ poses to be optimized sequentially and $\mathbf{T}_{n}$ is the last pose similar with $\mathbf{T}_{\mathrm{L}}$ in Fig. 4 . For the $i$ th pose $\mathbf{T}_{i}=\left\{\boldsymbol{\theta}_{i}, \mathbf{t}_{i}\right\}, i \in[2, n]\left(\mathbf{T}_{1}\right.$ does not need to be corrected), the adjustment of 3-DoF positions is described as

$$
\tilde{\mathbf{t}}_{i}=\mathbf{t}_{i}+\left\|\mathbf{t}_{i}-\mathbf{t}_{i-1}\right\| \mathbf{t}_{\mathrm{err}} / \sum_{k=2}^{n}\left\|\mathbf{t}_{k}-\mathbf{t}_{k-1}\right\|
$$

where $\tilde{\mathbf{t}}_{i}$ is the corrected position of $\mathbf{t}_{i}$ and $\mathbf{t}_{\text {err }}$ is the $3-\mathrm{DoF}$ position error of the final gap.

The 3-DoF orientations are adjusted after the former adjustment of 3-DoF positions. The orientation error $\boldsymbol{\theta}_{\text {err }}$ occurs due to the change of positions from $\mathbf{t}_{i}$ to $\tilde{\mathbf{t}}_{i}$ in the former step, which can be calculated by registering $\mathbf{t}_{1}, \ldots, \mathbf{t}_{n}$ and $\tilde{\mathbf{t}}_{1}, \ldots, \tilde{\mathbf{t}}_{n}$ using the iterative closest point (ICP) algorithm [19]. The adjustment of 3-DoF orientations can be described as

$$
\Delta \boldsymbol{\theta}_{i}=\tilde{\theta}_{i}-\boldsymbol{\theta}_{i}=\left\|\tilde{\mathbf{t}}_{i}-\tilde{\mathbf{t}}_{i-1}\right\| \boldsymbol{\theta}_{\mathrm{err}} / \sum_{k=2}^{n}\left\|\tilde{\mathbf{t}}_{k}-\tilde{\mathbf{t}}_{k-1}\right\|
$$

where $\Delta \boldsymbol{\theta}_{i}$ is the changing orientation for the $i$ th pose. In practice, we find that the main variation exists in yaw angles and only the yaw angle is used in $\Delta \boldsymbol{\theta}_{i}$ for convenience. Finally, the corrected poses $\tilde{\mathbf{T}}_{i}=\left\{\tilde{\theta}_{i}, \tilde{\mathbf{t}}_{i}\right\}$ are used as initial values for the graph optimization of iSAM2 and the Drift Error Correction module outputs data of poses and point clouds with no drift.

\section{B. GNSS-LiDAR Mapping}

The implementation details of the GL-mapping algorithm for GNSS-LiDAR mapping mode are presented in this section. GL-mapping maintains a LiDAR data registration method (i.e., the GNSS-initialized LiDAR registration module) derived from the LOAM algorithm to estimate positions as well as orientations. The GNSS RTK positioning is applied as the prior 3-DoF position and the reference coordinate system for the LiDAR data registration, while the result poses of LiDAR data registration are then used to align the coordinate systems between LiDAR and GNSS (i.e., the auto coordinate alignment module). The loosely coupled manner of GNSS RTK positioning and the LiDAR data registration have the advantages of: 1) the final estimated poses have no drift with the constraint of GNSS RTK positioning and 2) the orientations can be estimated without accurate orientation measurements from hardware systems.

The alignment of coordinate systems between GNSS and LiDAR means to estimate the transformation $\mathbf{T}_{L 0}^{W}$ from the first LiDAR coordinate $\left\{L_{0}\right\}$ to $\{W\}$, which cannot be measured without hardware systems providing absolute orientation measurements (e.g., GNSS dual-antenna). The auto coordinate alignment module performs an online calibration step to estimate $\mathbf{T}_{L 0}^{W}$ by iteratively registering two sets of 3-DoF positions: $\mathcal{U}^{W}$ from the final estimated poses and $\mathcal{V}^{W}$ from GNSS RTK positioning. The positions from LiDAR-SLAM (i.e., the LOAM algorithm) are added to $\mathcal{U}^{W}$ at the initial phase when there are no estimated poses yet. 
The local shapes of two trajectories (i.e., two sets of position data) of $\mathcal{U}^{W}$ and $\mathcal{V}^{W}$ are almost the same. However, the two trajectories do not coincide due to the transformation between them. As 3D-M-Box moves in the open scenes, two trajectories of $\mathcal{U}^{W}$ and $\mathcal{V}^{W}$ tend to coincide by the iterative registrations between them, which results in the convergency of $\mathbf{T}_{L 0}^{W}$. For the registration method, the sample consensus initial alignment (SAC-IA) [20] algorithm is applied to accomplish coarse registration for the reason that it is not sensitive with the initial values. Furtherly, the result from SAC-IA can be used as initial parameters for the ICP registration with high accuracy. The registration between $\mathcal{U}^{W}$ and $\mathcal{V}^{W}$ runs iteratively for every $k_{r}$ frames of LiDAR data and terminates when the estimated $\mathbf{T}_{L 0}^{W}$ changes slightly or maximum iterations are reached.

With the unified coordinate system, the GNSS-initialized LiDAR registration module utilizes an optimization step to estimate the 6-DoF pose of ego-motion. The optimization in this module derives from the LOAM algorithm, which is known as the combination of the Scan Registration, LiDAR odometry, and the LiDAR mapping [7]. However, the LiDAR odometry in LOAM is abandoned as it only provides a coarse pose estimation for LiDAR mapping and can be replaced by the GNSS RTK positioning data. The 3-DoF positions from GNSS RTK positioning are set as the initial values of the Levenberg-Marquardt (LM) optimization step in the LiDAR mapping with high accuracy. The 3-DoF orientations inferred by the relative increment of IMU measurements are also applied to the initialization (rather than initial orientations with zero). It should be noted that the Scan Registration module of the LOAM algorithm is reserved to deal with the point cloud distortion and perform feature points extraction.

The GNSS-initialized LiDAR registration finally outputs the real-time poses $\mathbf{T}^{W}$ and corresponding point clouds $P^{W}$ with high accuracy. The newly outputted $\mathbf{T}^{W}$ is then used for updating $\mathbf{T}_{L 0}^{W}$ in next iteration. In practice, the 3-DoF positions estimated by the GNSS-initialized LiDAR registration are nearly identical with the GNSS RTK measurements after $\mathbf{T}_{L 0}^{W}$ has converged. We only use the estimated 3-DoF orientations in final $\mathbf{T}^{W}$, while the 3-DoF positions are achieved directly from the GNSS RTK measurements. The details of the GL-mapping algorithm can be seen in Algorithm 1.

\section{EXPERIMENTAL RESULTS}

\section{A. Experiment Setup and Ground Truth}

Experiments are conducted to test the accuracy of the pose estimation results for the proposed algorithms of the two mapping modes, respectively. All the algorithms are implemented using $\mathrm{C}++$ language and can be achieved online. 3D-M-Box is applied in the experiments to acquire multisensor data and run the proposed algorithms. As is shown in Fig. 5, 3D-M-Box is mounted on a large-size unmanned ground vehicle (UGV) which is convenient for performing experiments in large-scale outdoor environments although the algorithms and the hardware system in this work are designed to be suitable for portable platforms. The speed of the UGV is about $1-2 \mathrm{~m} / \mathrm{s}$ according to the road conditions while working.
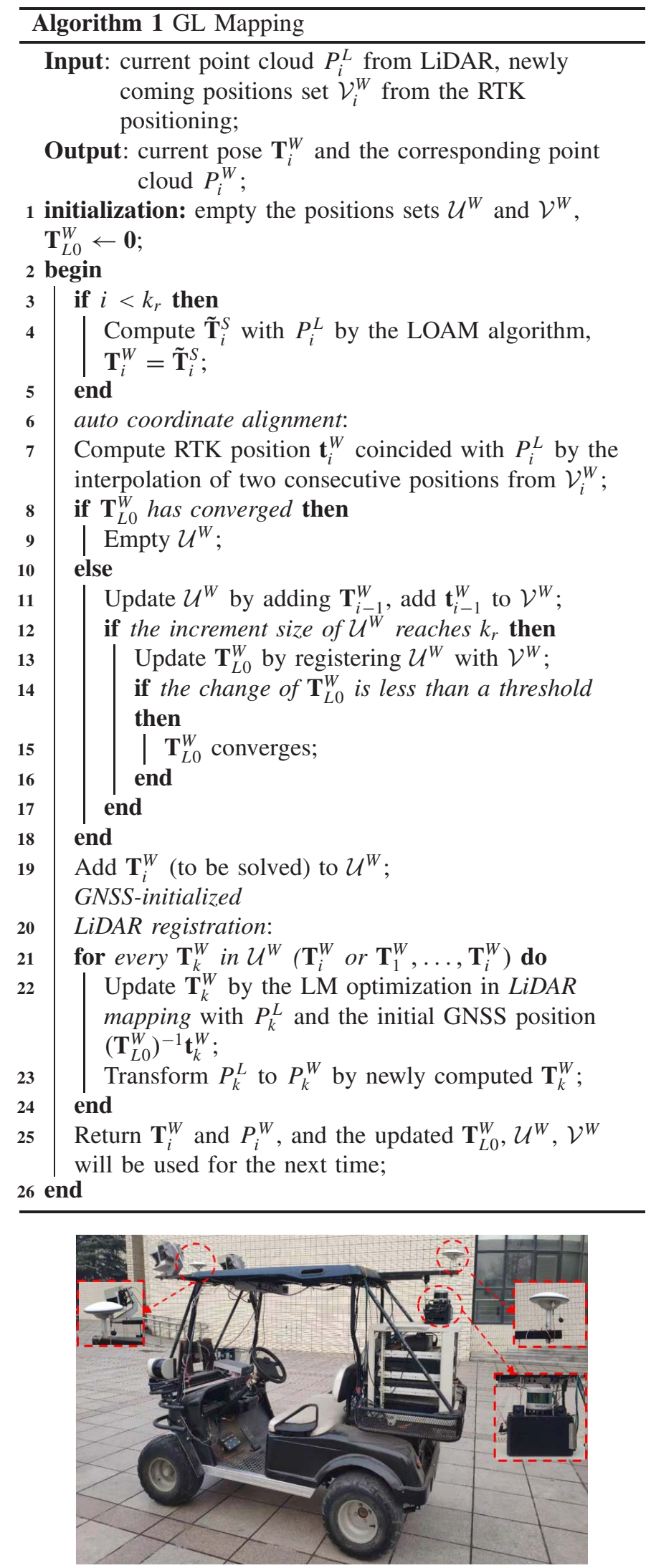

Fig. 5. 3D-M-Box is mounted on a UGV with GNSS dual-antenna in our experiments.

The use of a large-size UGV can deploy a GNSS dual-antenna with a long baseline so that the ground truth of orientations can be obtained with high accuracy. The ground 


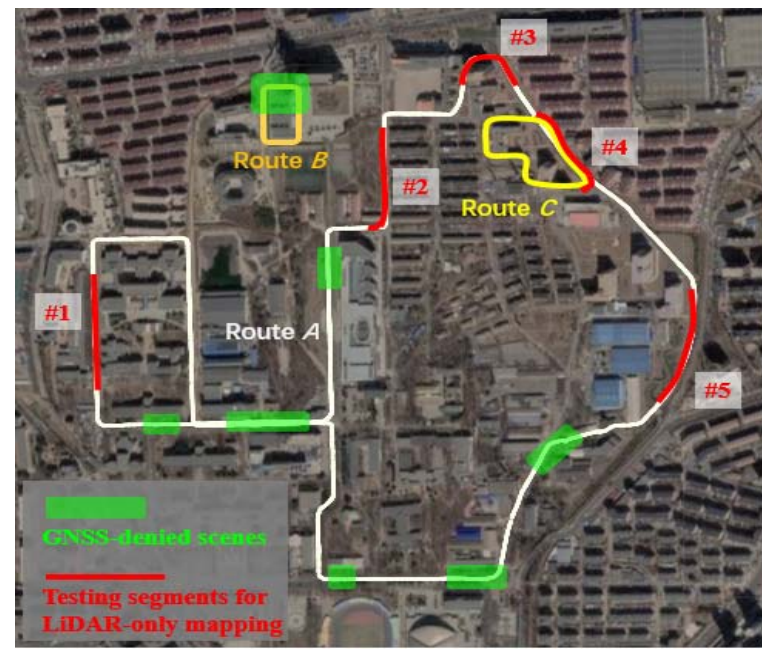

Fig. 6. Illustration of the testing sites in DUT campus.

truth of poses $\overline{\mathbf{T}}^{W}=\left(\overline{\boldsymbol{\theta}}^{W}, \overline{\mathbf{t}}^{W}\right)$ in this work is acquired by the 2-DoF orientation measurements from GNSS dual-antenna (only yaw and pitch can be measured by the GNSS dualantenna) and the 3-DoF position measurements from RTK positioning. As shown in Fig. 5, the baseline of the dualantenna is more than $2 \mathrm{~m}$. According to the user manual of the GNSS board in 3D-M-Box, the measurement error of the 2-DoF orientation is less than $0.05 .^{\circ}$ (with the $2-\mathrm{m}$ baseline), while the measurement error of the 3-DoF positions is less than $2 \mathrm{~cm}$ when RTK positioning is available. The only criterion confirming an effective RTK positioning is that the output NMEA sentences (i.e., GPGGA in this work) of the GNSS board have the flag of fixed solution or free solution; otherwise, the RTK positioning is unavailable.

It should be noticed that the measurements from GNSS dual-antenna are only used as the ground truth of orientations and not used in the proposed mapping framework. However, we do not have ground truth of poses for the proposed approach in the LiDAR-only mapping mode as the RTK positioning is unavailable. In order to evaluate the accuracy of the estimated poses, random segments of scenes along the experimental trajectory are chosen by hand, where the RTK positioning is available, but the RTK positioning data are blocked for the proposed algorithm by software technique.

\section{B. Testing Sites}

The varieties of partially GNSS-denied outdoor environments differ by the sparse/dense degree of the distribution of landscapes (e.g., buildings, trees, etc.), which affects the effectiveness of RTK positioning and the accuracy of LiDARbased pose estimation algorithms. We drive the UGV equipped with 3D-M-Box in the Dalian University of Technology (DUT) campus to achieve large-scale testing data for various kinds of outdoor environments. As shown in Fig. 6, three routes are chosen for the data acquisition. Route $A$ (with white color in Fig. 6) covers most of the trunk roads in the campus and results in a large-scale urban testing site with a long distance of $5 \mathrm{~km}$, where various kinds of buildings, trees, cars, and artificial landscape exist. Another two testing routes are designed along the particular pathway to consider more extreme environments. Route $B$ (with dark yellow in Fig. 6) covers a narrow path with a length of $300 \mathrm{~m}$, which encircles a strip-shape building with a short corridor. The width of the path (i.e., the distance between the building and the tall wall/trees on the other side) is only 3-6 m. Route $C$ (with bright yellow in Fig. 6) has a length of $500 \mathrm{~m}$ and is full of open space, where most of the buildings are more than $40 \mathrm{~m}$ away from the road.

It was found that the RTK positioning is available in most of the scenes for the typical urban environments like Route $A$, but will lose effectiveness for a short distance (i.e., tens of meters) at several places where the occlusion of the buildings/trees is dense. However, nearly half of the trajectory for Route $B$ is composed of GNSS-denied scenes due to the occlusion, while all the scenes in Route $C$ are open scenes. The usage of different experimental routes is as follows:

1) Random segments of scenes along with Routes $A$ and $C$ (\#1-\#5) are chosen to test the pose estimation accuracy of the proposed algorithms in LiDAR-only mapping mode, where the RTK positioning is available but will be blocked for the algorithms.

2) All the scenes in Routes $A-C$ with effective RTK positioning are used to test the pose estimation accuracy of the GL-mapping algorithm in GNSS-LiDAR mapping mode. More specifically, Route $C$ helps to test the accuracy in relatively open areas with sparse landscapes.

3) The final trajectories and point clouds of Routes $A-C$ are given to furtherly demonstrate the overall feasibility of proposed algorithms for various kinds of outdoor environments. All the above details can be seen in Fig. 6 .

\section{Pose Estimation Accuracy of LiDAR-Only Mapping}

Five segments are randomly chosen from the experimental trajectories illustrated in Fig. 6, where the 5-DoF pose ground truth is available (except the roll angle) but blocked in the algorithms of LiDAR-only mapping mode. The results of the pose estimation accuracy are shown in Table I. To make the results more convincing, the distance of every selected segment is longer than $100 \mathrm{~m}$ although it is generally tens of meters for the distance of GNSS-denied segments in real-world cases. It can be seen from the last two rows of Table I that the proposed algorithm with the Drift Error Correction module of this work can eliminate the drift errors to the most extent, especially for the position errors on the $z$-axis. An example of the correction of drift errors (segment \#4 in Route $C$ ) is shown in Fig. 7.

\section{Pose Estimation Accuracy of GNSS-LiDAR Mapping}

The proposed loosely coupled GL-mapping algorithm in GNSS-LiDAR mapping mode primarily estimates the 3-DoF orientations with the help of the 3-DoF positions from GNSS RTK positioning data. Although GL-mapping can be set up to output its own estimated 3-DoF positions, the RTK positions as well as the estimated orientations are used to construct the final 6-DoF poses for 3-D map building. This manner of the construction of the 6-DoF poses depends on the fact that 


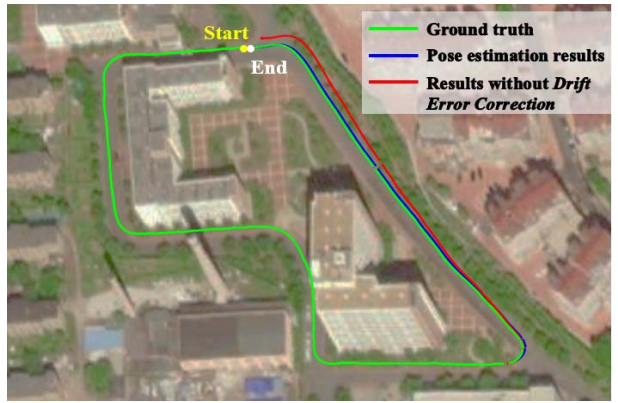

Fig. 7. Example result of drift error correction (segment \#4 in Route C).

TABLE I

Mean Pose Estimation ERrors

\begin{tabular}{|c|c|c|c|c|c|c|}
\hline $\begin{array}{l}\text { Segment } \\
\text { No. }\end{array}$ & $\begin{array}{l}\text { Distance } \\
(\mathrm{m})\end{array}$ & $\begin{array}{c}\mathbf{X} \\
(\mathbf{m})\end{array}$ & $\begin{array}{c}\mathbf{Y} \\
(\mathbf{m})\end{array}$ & $\begin{array}{c}\mathbf{Z} \\
(\mathbf{m})\end{array}$ & $\begin{array}{l}\text { Yaw } \\
(\mathrm{rad})\end{array}$ & $\begin{array}{l}\text { Pitch } \\
\text { (rad) }\end{array}$ \\
\hline$\# 1$ & 119.06 & 0.22 & 0.26 & 0.59 & 0.0257 & 0.0272 \\
\hline \#2 & 132.15 & 0.37 & 0.21 & 0.09 & 0.0103 & 0.0115 \\
\hline \#3 & 143.07 & 0.16 & 0.22 & 0.56 & 0.0252 & 0.0194 \\
\hline \#4 & 177.48 & 0.16 & 0.28 & 0.47 & 0.0025 & 0.0295 \\
\hline$\# 5$ & 133.95 & 0.40 & 0.48 & 0.14 & 0.0001 & 0.0249 \\
\hline \multicolumn{2}{|c|}{ Average results } & 0.26 & 0.29 & 0.37 & 0.0128 & 0.0225 \\
\hline \multicolumn{2}{|c|}{$\begin{array}{l}\text { Results without Drift } \\
\text { Error Correction }\end{array}$} & 0.94 & 0.55 & 3.64 & 0.0149 & 0.0292 \\
\hline
\end{tabular}

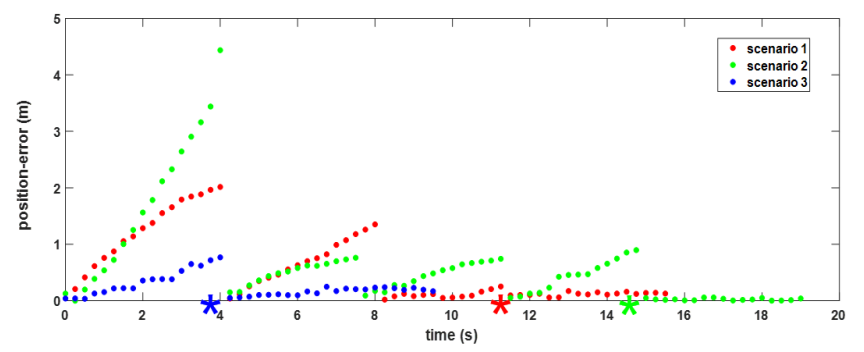

Fig. 8. Three experimental segments of position errors between the estimated positions of GL-mapping and the positions of RTK measurements. The position errors converge to zero as $\mathbf{T}_{L 0}^{W}$ converges. The asterisk represents the time when corresponding $\mathbf{T}_{L 0}^{W}$ converges.

the estimated 3-DoF positions of GL-mapping will converge to RTK positions along with the online calibration of $\mathbf{T}_{L 0}^{W}$.

Experimental results are given to explain the convergence of the estimated positions of GL-mapping. As shown in Fig. 8, three example segments are selected randomly from the experimental trajectories. The position errors between the estimated positions of GL-mapping and the positions of RTK measurements for each selected segment are provided and illustrated by different colors. It should be noted that at the beginning of GNSS-LiDAR mapping mode, GL-mapping maintains a started position as same as the RTK position and the initial position error is zero.

However, the position errors grow almost linearly due to different coordinate systems between GNSS and LiDAR. The slope of every trajectory in Fig. 8 positively correlated with the current orientation difference (i.e., the rotational difference between current estimated $\mathbf{T}_{L 0}^{W}$ and the ground truth of $\mathbf{T}_{L 0}^{W}$ ) to be determined for the two coordinate systems. For every $k_{r}$

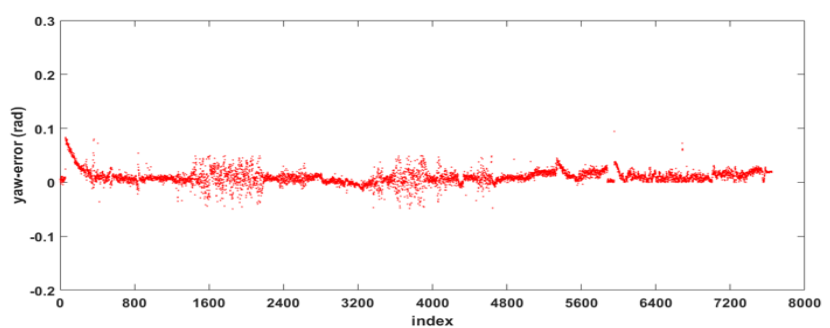

(a)

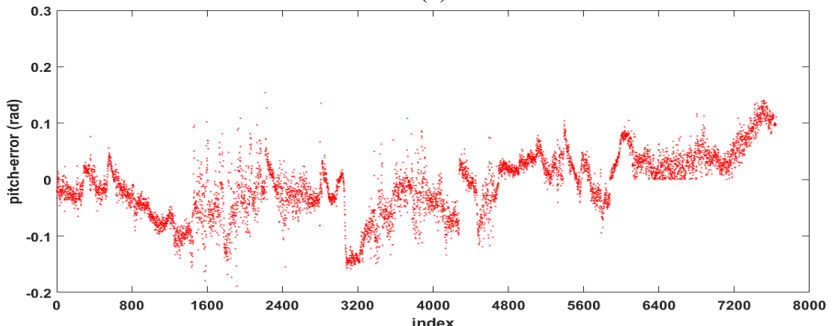

(b)

Fig. 9. Statistics of the rotational errors of (a) yaw and (b) pitch in the GNSS-LiDAR mapping mode.

(which is set as 15 in the experiment) frames of LiDAR data, GL-mapping runs once and $\mathbf{T}_{L 0}^{W}$ is reestimated. Then, $\mathbf{T}_{L 0}^{W}$ contributes to the pose estimation so that the position errors are suppressed to some extent. In practice, the translational part of $\mathbf{T}_{L 0}^{W}$ can be estimated with high accuracy, so that the position error can return to zero in the figure for every execution and result in a piecewise curve. However, the convergence of the rotational part of $\mathbf{T}_{L 0}^{W}$ was found to need several times of execution. The position errors finally converge to zero after the convergence of $\mathbf{T}_{L 0}^{W}$. The procedure of the convergence usually finishes within seconds.

The average position errors after convergence of $\mathbf{T}_{L 0}^{W}$ of all the experimental segments in the GNSS-LiDAR mapping mode are about $0.03 \mathrm{~m}$. The results prove the feasibility of the online calibration of $\mathbf{T}_{L 0}^{W}$ and the coincidence between the estimated poses of GL-mapping and the RTK positioning. The RTK positions can be directly derived to construct the final 6-DoF poses for 3-D map building.

The accuracy of the estimated 2-DoF orientations (except the roll angle) of GL-mapping is then evaluated. The statistics of the rotational errors for yaw and pitch are shown in Fig. 9, which are gathered among all the open scenes of Routes $A-C$. There are almost 8000 estimated poses that are sequentially shown in the figure (a few poses are abandoned whose corresponding ground truth angles are obviously outliers due to the unstable hardware measurements). The average rotational error of yaw is $0.0120 \mathrm{rad}$ and that of pitch is $0.0445 \mathrm{rad}$. The results validate that the proposed algorithm can estimate accurate orientations without the support of the high-cost inertial device or the GNSS dual-antenna. More specifically, the average rotational errors of Route $C$ are 0.0143/0.0494 rad for the yaw/pitch, which proves the feasibility in relatively open areas with sparse landscapes.

\section{E. Overall Results of Pose Estimation and Map Building}

The trajectories of estimated poses and corresponding point clouds of Routes $A-C$ are illustrated in Fig. 10, which 


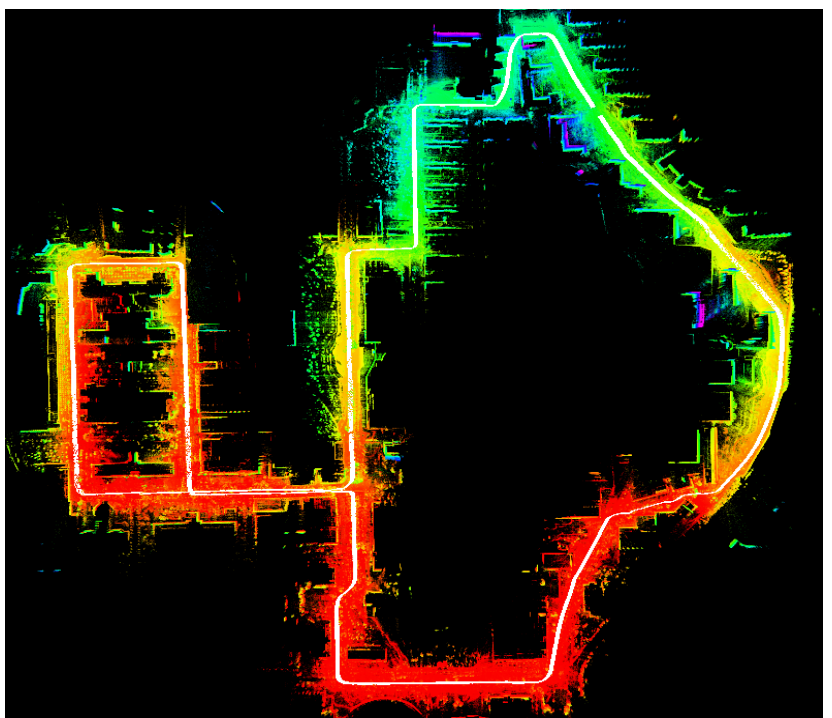

(a)

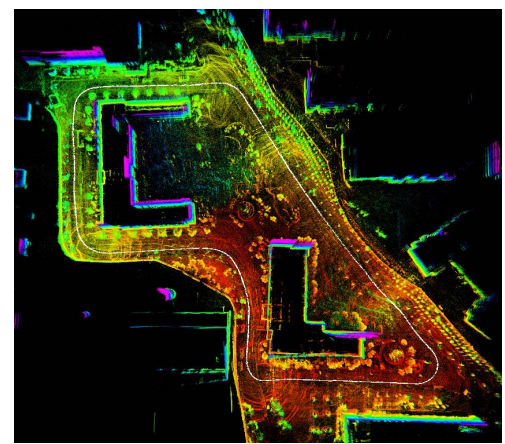

(b)

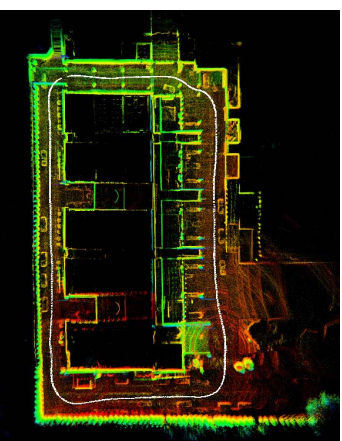

(c)

Fig. 10. Trajectories of estimated poses and corresponding point clouds of (a) Route $A$, (b) Route $B$, and (c) Route $C$. The trajectories are in white color. The color of point clouds is differed by elevation (i.e., along the $z$-axis).

helps to visually demonstrate the overall feasibility of the proposed framework in various kinds of outdoor environments. It should be noted that the map building results are obtained by transforming raw LiDAR data into global coordinate with the corresponding estimated poses. Other factors that affect the quality of the map building (e.g., the ranging accuracy of the LiDAR sensor) are not concerned in this work.

\section{F. Discussion}

The accuracy of the estimated roll angles is not evaluated due to the lack of ground truth. However, the roll angle changes slightly during the testing data acquisition with the large-size UGV and its accuracy can be inferred to be acceptable according to the evaluation of the other two angles. The experimental results validate that the estimated poses have no drift errors with the constraint of RTK positioning.

Indeed, the property of the proposed pose estimation framework is similar to that of a GNSS/INS system in several points. They all provide consistent poses for a short period of time when GNSS is unlocked. However, the main differences are as follows:

1) The proposed framework and the hardware system of this work only focus on the map building and can eliminate the drift errors of the unlocking period.
2) Apart from the LiDAR sensor, the proposed mapping framework only needs a GNSS and an optional IMU to estimate poses, while the GNSS/INS-based system needs the combination of GNSS, INS, and dual-antenna for most of the applications.

In conclusion, the proposed mapping system of this work maintains a lightweight hardware component with acceptable pose estimation accuracy.

Besides, the proposed pose estimation framework is not an SLAM system. The classic SLAM framework and GNSS positioning are integrated into the proposed framework and the only usage of the estimated poses is to perform map building. Furthermore, it is generally only tens of meters for the distance of GNSS-denied segments in real-world cases, so that the typical problem of loop closure in LiDAR SLAM does not need to be concerned.

Although the time efficiency is generally not important for a pose estimation framework aiming at map building, the proposed framework in this article is almost real-time except two time-delayed steps: the Drift Error Correction module in LiDAR-only mapping mode and the update of poses before the convergence of $\mathbf{T}_{L 0}^{W}$ in GNSS-LiDAR mapping mode. For the experimental segments in Table I, the average length of one segment is about $140 \mathrm{~m}$. It takes about $710 \mathrm{~ms}$ in average for the Drift Error Correction module to correct drift errors and transform point clouds for every segment. The second time-delayed step lasts from 10 to $200 \mathrm{~ms}$ according to the degree of the convergence of $\mathbf{T}_{L 0}^{W}$, which usually lasts for seconds (as shown in Fig. 8). Other main modules in the framework are close to real time for the reason that their structures are derived from the real-time LOAM algorithms. All the time consumption results are achieved by the execution on the micro-computer in 3D-M-Box. It should be noted that the time-delayed steps can be abandoned (i.e., the accuracy of the pose estimation is sacrificed for some scenes) so that the proposed framework can be thoroughly real time for applications aiming at online localization (e.g., the navigation of mobile robots).

\section{CONClusion}

This article has focused on the development of a mobile mapping system that can operate in large-scale outdoor environments. An integrated GNSS/LiDAR-SLAM pose estimation framework is proposed to accomplish 3-D map building in partially GNSS-denied environments. Experimental results show the validity and performance of the proposed system and algorithms. In our future work, the fusion between laser point clouds and vision data will be investigated, which is the fundamental function for semantic map building and scene understanding.

\section{REFERENCES}

[1] L. Wei, C. Cappelle, and Y. Ruichek, "Camera/laser/GPS fusion method for vehicle positioning under extended NIS-based sensor validation," IEEE Trans. Instrum. Meas., vol. 62, no. 11, pp. 3110-3122, Nov. 2013.

[2] Z. Gong, C. Wen, C. Wang, and J. Li, "A target-free automatic self-calibration approach for multibeam laser scanners," IEEE Trans. Instrum. Meas., vol. 67, no. 1, pp. 238-240, Jan. 2018. 
[3] D. A. Grejner-Brzezinska, "Mobile mapping technology: Ten years later-Part I," Surveying Land Inf. Syst., vol. 61, no. 2, pp. 79-94, 2001.

[4] D. A. Grejner-Brzezinska, "Mobile mapping technology: Ten years later-Part II," Surveying Land Inf. Syst., vol. 61, no. 3, pp. 83-100, 2001.

[5] D. Grejner-Brzezinska, C. Toth, and Y. Yi, "On improving navigation accuracy of GPS/INS systems," Photogramm. Eng. Remote Sens., vol. 71, no. 4, pp. 377-389, Apr. 2005.

[6] W. Hess, D. Kohler, H. Rapp, and D. Andor, "Real-time loop closure in 2D LIDAR SLAM," in Proc. IEEE Int. Conf. Robot. Autom. (ICRA), May 2016, pp. 1271-1278.

[7] J. Zhang and S. Singh, "Low-drift and real-time lidar odometry and mapping," Auto. Robots, vol. 41, no. 2, pp. 401-416, Feb. 2017.

[8] T. Shan and B. Englot, "LeGO-LOAM: Lightweight and groundoptimized lidar odometry and mapping on variable terrain," in Proc. IEEE/RSJ Int. Conf. Intell. Robots Syst. (IROS), Oct. 2018, pp. 4758-4765.

[9] H. Ye, Y. Chen, and M. Liu, "Tightly coupled 3D lidar inertial odometry and mapping," in Proc. Int. Conf. Robot. Autom. (ICRA), May 2019, pp. 3144-3150.

[10] T. Shan, B. Englot, D. Meyers, W. Wang, C. Ratti, and R. Daniela, "LIO-SAM: Tightly-coupled lidar inertial odometry via smoothing and mapping," in Proc. IEEE/RSJ Int. Conf. Intell. Robots Syst. (IROS), Jul. 2020, pp. 1-8.

[11] H. Lauterbach, D. Borrmann, R. Heß, D. Eck, K. Schilling, and A. Nüchter, "Evaluation of a backpack-mounted 3D mobile scanning system," Remote Sens., vol. 7, no. 10, pp. 13753-13781, Oct. 2015.

[12] Y. Su, H. Guan, T. Hu, and Q. Guo, "The integration of uavand backpack LiDAR systems for forest inventory," IEEE Int. Geosci. Remote Sens. Symp., Jul. 2018, pp. 8757-8760.

[13] D. Zhang, Z. Gong, Y. Chen, J. Zelek, and J. Li, "SLAM-based multi-sensor backpack lidar systems in GNSS-denied environments," in Proc. IEEE Int. Geosci. Remote Sens. Symp. (IGARSS), Jul. 2019, pp. 8984-8987.

[14] D. A. Grejner-Brzezinska, C. K. sToth, H. Sun, X. Wang, and C. Rizos, "A robust solution to high-accuracy geolocation: Quadruple integration of GPS, IMU, pseudolite, and terrestrial laser scanning," IEEE Trans. Instrum. Meas., vol. 60, no. 11, pp. 3694-3708, Nov. 2011.

[15] S. Chen and C. Chen, "Probabilistic fuzzy system for uncertain localization and map building of mobile robots," IEEE Trans. Instrum. Meas., vol. 61, no. 6, pp. 1546-1560, Jun. 2012.

[16] C. Qian et al., "An integrated GNSS/INS/LiDAR-SLAM positioning method for highly accurate forest stem mapping," Remote Sens., vol. 9, no. 1, p. 3, Dec. 2016.

[17] A. V. Kanhere and G. X. Gao, "Integrity for GPS/LiDAR fusion utilizing a RAIM framework," in Proc. 31st Int. Tech. Meeting Satell. Division Inst. Navigat. (ION GNSS), Oct. 2018, pp. 3145-3155.

[18] M. Kaess, H. Johannsson, R. Roberts, V. Ila, J. J. Leonard, and F. Dellaert, "ISAM2: Incremental smoothing and mapping using the Bayes tree," Int. J. Robot. Res., vol. 31, no. 2, pp. 216-235, Feb. 2012.

[19] A. V. Segal, D. Haehnel, and S. Thrun, "Generalized-ICP," Robot. Sci., Syst., vol. 2, no. 4, p. 435, Jun. 2009.

[20] R. B. Rusu, N. Blodow, and M. Beetz, "Fast point feature histograms (FPFH) for 3D registration," in Proc. IEEE Int. Conf. Robot. Autom., May 2009, pp. 1848-1853.

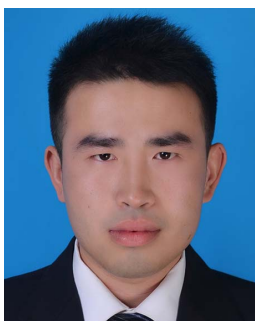

Guojian He received the bachelor's and master's degrees in control theory and engineering from the Dalian University of Technology, Dalian, China, in 2011 and 2014, respectively, where he is currently pursuing the Ph.D. degree with the School of Control Science and Engineering.

His research interests include mobile robot navigation, 3-D LiDAR SLAM, mapping, and semantic scene understanding.

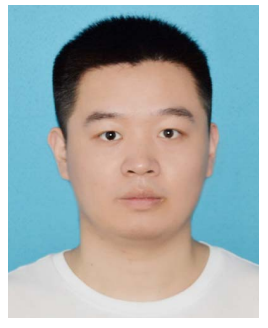

Xingda Yuan received the bachelor's degree from the School of Control Science and Engineering, Dalian University of Technology, Dalian, China, in 2018, where he is currently pursuing the master's degree.

His research interests include 3-D LiDAR SLAM, mapping, and localization.

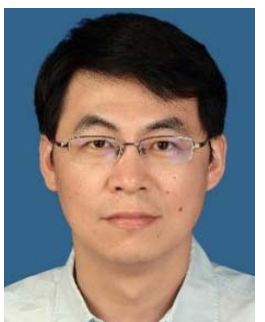

Yan Zhuang (Member, IEEE) received the bachelor's and master's degrees in control theory and engineering from Northeastern University, Shenyang, China, in 1997 and 2000, respectively, and the Ph.D. degree in control theory and engineering from the Dalian University of Technology, Dalian, China, in 2004.

In 2005, he joined the Dalian University of Technology as a Lecturer and became an Associate Professor in 2007. Currently, he is a Professor with the School of Control Science and Engineering, Dalian University of Technology. His research interests include mobile robot 3-D mapping, 3-D scene recognition, and reconstruction.

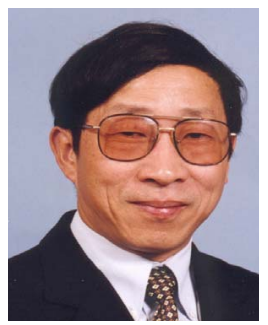

Huosheng Hu (Senior Member, IEEE) received the M.Sc. degree in industrial automation from Central South University, Changsha, China, in 1982, and the $\mathrm{Ph} . \mathrm{D}$. degree in robotics from the University of Oxford, Oxford, U.K., in 1993.

$\mathrm{He}$ is a Professor with the School of Computer Science and Electronic Engineering, University of Essex, Colchester, U.K., where he is leading the Robotics Research Group. He has published around 450 articles in journals, books, and conferences in these areas. His research interests include behavior-based robotics, human-robot interaction, service robots, embedded systems, data fusion, learning algorithms, mechatronics, and pervasive computing.

Dr. Hu received a number of best letter awards. He is a founding member of the IEEE Robotics and Automation Society Technical Committee on Networked Robots, a fellow of the Institution of Engineering and Technology (IET) and Institute of Measurement and Control (InstMC), and a Senior Member of the Association for Computing Machinery (ACM). Currently, he serves as the Editor-in-Chief for International Journal of Automation and Computing and online Robotics Journal, and the Executive Editor for International Journal of Mechatronics and Automation. 